\title{
Sedimentforschung und -management
}

Fließgewässer sind natürlich vom Wasserdurchfluss und dessen Variabilität geprägt und können dahingehend auch charakterisiert werden. Gleichzeitig sind Flüsse Erosions- und Umlagerungserscheinungen, deren Ausformung stark vom Sedimenttransport abhängig ist. Je nach Sedimentdargebot im Vergleich zur Transportkapazität, verfügbaren Korngrößen, räumlichen Randbedingungen oder auch Vegetationseinfluss bilden sich verschiedene Flusstypen aus. Natürliche und anthropogene Änderungen beeinflussen diesen Systemzustand. So kann der Klimawandel aufgrund des Gletscherschwunds oder Rückgangs des Permafrosts zu einem erhöhten Sedimenteintrag führen, ebenso Landnutzungsänderungen wie intensivierte Landwirtschaft. Andererseits kommt es durch Querbauwerke wie Wasserkraftanlagen im Staubereich zu Sedimentüberschuss während in freien Fließstrecken flussab Sedimentdefizit vorherrscht. Das Sedimentdefizit führt vielfach zu Sohleintiefungen mit damit verbundenen Instabilitäten der Ufersicherungen und folgender Erhöhung des Hochwasserrisikos, Verlust an gewässertypischen Strukturen und Verschlechterungen des ökologischen Zustands, wenn z. B. kein Laichkies mehr vorhanden ist. Jüngste Untersuchungen an der Donau zeigten, dass die Sedimentbilanz völlig gestört ist, mit Überschuss- und Defizitstrecken, wo schließlich von den ursprünglich ca. 50 Mio.t Schwebstoffen pro Jahr nur mehr weniger als die Hälfte ins Schwarze Meer transportiert werden. Die Folge ist eine Küstenerosion von bis zu $24 \mathrm{~m} / J a h r$. In den Überschussstrecken der Flüsse besteht das Problem, dass z. B. aus Hochwasserschutz- oder betriebstechnischen Gründen gebaggert werden muss und dieses Material möglicherweise dann mit gesamten Kosten von derzeit ca. 10 bis $20 € / \mathrm{m}^{3}$ zu deponieren ist.

Daraus folgt, dass Sedimente technisch, wirtschaftlich und ökologisch von zentraler Bedeutung für das Fließgewässermanagement sind.

Sedimentforschung und -management sind im Wasserbau daher zunehmend wichtiger, um eine nachhaltige Entwicklung der Flüsse und deren Nutzung zu ermöglichen. Dies umfasst die Wildbach- und Lawinenverbauung, die Bundeswasserbauverwaltung, die Energiewirtschaft, die Wasserstraße, die Ökologie aber auch die Land- und Forstwirtschaft.

In diesem Heft der Österreichischen Wasser- und Abfallwirtschaft behandeln neun Artikel das Thema Sedimentforschung und -management aus verschiedenen Blickwinkeln.

Habersack et al. stellen internationale Aktivitäten in Zusammenhang mit Sedimentforschung und -management auf globaler, europäischer, regionaler und Einzugsgebietsebene vor. Auf globaler Ebene gibt es Aktivitäten bei der UNESCO wie die World's Large Rivers Initiative oder bei der World Association for Sedimentation and Erosion Research WASER. SedNet ist eine europäische Initiative, die Sedimentforschung und -management in Europa vorantreibt. Schließlich findet man in den Einzugsgebieten der Donau, des Rheins und der Elbe zunehmend Aktivitäten zum Sedimentmanagement.

Herausforderungen des Feststoffmanagements bei Wasserkraftanlagen in Österreich behandeln Bock et al. Während die Veränderung des Feststoffhaushalts durch die Wasserkraftnutzung unvermeidlich ist, zeigen die Autorlnnen, dass jedoch konstruktive Maßnahmen bei der Kraftwerksplanung und eine an die natürlichen Verhältnisse angepasste Betriebsweise diese reduzieren können. Der Artikel beschreibt anhand von Fallbeispielen Methoden des Feststoffmanagements und Anforderungen an die Forschung, insbesondere in Hinblick auf das Prozessverständnis. Sie verweisen dabei auf das Christian Doppler Labor für Sedimentforschung und -management.

Hauer et al. stellen das Christian Doppler Labor für Sedimentforschung und -management vor. Dieses wird in enger Zusammenarbeit mit der Energiewirtschaft Österreichs, Turbinenherstellern und der Wasserstraßenverwaltung betrieben. Das Christian Doppler Labor für Sedimentforschung und -management hat zum Ziel, (i) Möglichkeiten zur optimierten ökonomischen, technischen bzw. öko- 
logischen Nutzung der Wasserkraft, insbesondere Minimierung der Stauraumverlandung, (ii) zur Verbesserung des Sedimentmanagements bei Wasserstraßen und (iii) zur Verlängerung der Lebensdauer unterschiedlicher technischer Anlagenteile von Wasserkraftanlagen (z. B. Turbinen) zu erarbeiten. Wesentlich sind ein verbessertes Sedimentmanagement und die Reduktion der Kosten unter Einhaltung technischer und ökologischer Vorgaben.

Das Monitoring des Sedimenttransports in der Natur ist zentral für die Erforschung von Sedimenttransportprozessen und die Überprüfung und Verbesserung theoretischer Berechnungsansätze. Liedermann et al. zeigen anhand von verschiedenen Beispielen Forschungsergebnisse zu alpinen Sedimentprozessen unter Berücksichtigung extremer Ereignisse. Neben der Kurzvorstellung von Messmethoden erfolgt die Definition von Geschiebetransportprozesstypen und die Darstellung der Auswirkungen der Sedimentverfügbarkeit auf den Geschiebetransport und der Berechnung der Transportgeschwindigkeit von Geschiebe sowie der Untersuchung der Sammeleffizienz von Geschiebefangkörben im Forschungsgerinne der BOKU.

Die Forschung zum Thema Sedimenttransport benötigt zur systematischen Ableitung von Prozesszusammenhängen und mathematischen Beschreibungen wasserbauliche Modellversuche. Der Artikel von Sindelar et al. stellt neue Entwicklungen im wasserbaulichen Modellversuchswesen zum Sedimenttransport vor. Dabei geht es um neue messmethodische Entwicklungen wie die Weiterentwicklung von PIV und stereo-PIV zu tomo-PIV und tomo-PTV. Schließlich werden neue experimentelle Forschungsansätze wie zeitaufgelöste 3D-Messungen bei Bewegungsbeginn eines Einzelsteins vorgestellt. Beim Sedimenttransport und Modellversuchswesen kommt dem Maßstab eine große Bedeutung zu, weshalb großmaßstäbliche Versuche zum Sedimenttransport im BOKU-Forschungsgerinne bis zum Maßstab 1:1 und die Abschätzung der Skalierungsfehler im Fachbeitrag angesprochen werden.

Mathematische Beschreibungen des Sedimenttansports aus Theorie, Modellversuchen und Feldmessungen dienen als Grundlage für die numerische Simulation. Tritthart et al. diskutieren in ihrem Beitrag Erfahrungen in der numerischen Sedimenttransportmodellierung auf unterschiedlichen Skalen - von RANS bis LES. Im Sinne der Eulerschen Betrachtung wird das BOKU-Modell iSed vorgestellt, welches den Geschiebe- und Schwebstofftransport sowie die Morphodynamik 2D/3D für wissenschaftliche und praktische Anwendungen modellieren kann. Im Sinne der Lagrangeschen Betrachtung erfolgt die Diskussion eines numerischen Geschiebetracermodells. Auf kleiner Maßstabsebene wird die LargeEddy Simulation (LES) verwendet, wo u.a. die Grundlagenmodellversuche im Wasserbaulabor modelliert werden. Beispiele zu allen Skalen veranschaulichen die numerischen Simulationen.

Als Anwendungsbeispiel der verschiedenen Methoden und zur Lösung praktisch relevanter Problemstellungen beschreiben Rindler et al. ein innovatives Feststoffmanagement für Wildbacheinzugsgebiete am Beispiel des Strobler Weißenbaches. Maßnahmen zur Bewirtschaftung von Feststoffen wie Geschiebe und Wildholz können je nach Bautyp eine gänzliche Unterbrechung des Sedimentkontinuums bis hin zum temporären Rückhalt ergeben. Auf Basis von kontinuierlichen Abfluss- und Schwebstoffmessungen, der Bestimmung von Veränderungen des Sperrenvolumens mittels Drohnen, Messungen des Geschiebetransports mithilfe von RFID-Tracern etc. konnte der Erfolg der Maßnahmen (Entfernen von Totholz beim Sperrenschlitz und Sedimentzugabe) aus technischer Sicht nachgewiesen werden. In Bezug auf die Auswirkungen der Maßnahmen zeigte ein biotisches, fischökologisches Monitoring keine signifikanten Änderungen in der Biomasse der Bachforelle.

Ein weiteres Beispiel für praktisch relevante Themenstellungen zum Sedimentmanagement behandeln Brunner et al. Dabei geht es um die Optimierung des Sedimentmanagements zur Reduktion von Feinsedimentanlandungen in Gewässern des Weinviertels. Die Autorlnnen thematisieren dabei den flächenhaften Bodenabtrag aus landwirtschaftlich genutzten Gebieten im Zuge von Starkregenereignissen, die unerwünschte Anlandungen im Gewässerraum verursachen. Im Mittelpunkt stehen Fragen 


\title{
Editorial
}

wie: Wie wirken die landwirtschaftlich genutzten Flächen als diffuse Quelle der Sedimenteinträge? Welchen Anteil an problematischen Sedimentanlandungen hat das Fließgewässer als Transportsystem? Kann eine punktuelle bauliche Maßnahme lineare Gewässerstrecken von Anlandungen entlasten und damit den Räumungsbedarf auf einzelne Standorte einschränken?

Im neunten und letzten Artikel von Schobesberger et al. geht es um das Urbane Sedimentmanagement. Am Beispiel von Wienerwaldbächen erfolgt die Analyse der Sedimentherkunft und der gezielten Sedimentation, damit letztlich weniger Material in die Kanalisation der Stadt Wien eingetragen wird, das dort mitunter einen großen Räumaufwand verursacht. Die Beprobung des natürlichen und urbanen Einzugsgebiets ergab, dass ein Großteil der Sedimente aus dem städtischen Umfeld stammt. Weiters wurde ein existierender Schotterfang im BOKU-Wasserbaulabor physikalisch modelliert und analysiert. Es zeigte sich, dass nach einem simulierten Extremereignis nur ein kleiner Teil der Sedimente im Schotterfang bleibt und der Rest in den Kanal eingetragen wird. Zur Verbesserung der Wirkung konnten innovative bauliche Ansätze entwickelt werden, die im Vergleich zum IST-Zustand eine signifikante Verbesserung erzielen.

Abschließend sei noch erwähnt, dass es an der BOKU seit 1. Jänner 2019 das neue Institut für Wasserbau, Hydraulik und Fließgewässerforschung (IWA) gibt, welches neben anderen Forschungsfeldern als einen Schwerpunkt das Thema "Sedimente und Fließgewässer" behandelt. Dort ist auch das Christian Doppler Labor für Sedimentforschung und -management beheimatet. Mit dem Forschungsgerinne und künftigen neuen Wasserbaulabor stehen dem Institut und Kooperationspartnern wie dem Bundesamt für Wasserwirtschaft mit einem Freispiegelabfluss von bis zu $10 \mathrm{~m}^{3} / \mathrm{s}$ einzigartige Versuchsbedingungen zur Verfügung, um u. a. grundlagen- und anwendungsorientierte Forschung auf dem Gebiet des Sedimenttransports durchzuführen.

\author{
Univ.-Prof. DI Dr. H. Habersack ( $ه$ ) • \\ PD DI Dr. C. Hauer \\ Institut für Wasserbau, Hydraulik und \\ Fließgewässerforschung, Christian \\ Doppler Labor für Sedimentforschung \\ und -management, Department für \\ Wasser - Atmosphäre - Umwelt, \\ Universität für Bodenkultur Wien, \\ Muthgasse 107, \\ 1190 Wien, Österreich \\ helmut.habersack@boku.ac.at
}

